\title{
Paternally contributed centrioles exhibit exceptional persistence in C. elegans embryos
}

Cell Research (2015) 25:642-644. doi:10.1038/cr.2015.49; published online 24 April 2015

\section{Dear Editor,}

The two gametes make different contributions to the zygote at fertilization. Although both gametes contribute genetic material, in most animal species the oocyte donates the bulk of cytoplasmic constituents and cellular organelles, including mitochondria, whereas the sperm donates two centrioles. Centrioles are microtubule-based organelles that serve as templates for the axoneme of cilia and flagella across eukaryotic evolution, and as platforms for centrosome assembly in most animal cells [1]. How long the two centrioles contributed by the sperm persist in the developing embryo is not known in any system. More generally, although centrioles are reputed to be stable structures, the extent to which their constituents persist over several cell cycles has been scarcely studied. Two instances of centriolar components being stable for one ( $\alpha / \beta$ tubulin in mammalian cells) or two (SAS-4 in C. elegans) cell cycles have been reported [24], but whether these and other centriolar components remain stable for more cell cycles is not known.

We used $C$. elegans as a model system to assess the persistence of centriolar components in the context of a developing organism. We utilized a marked mating experimental strategy to specifically mark paternally contributed centriolar components and assess their persistence throughout embryonic development (Figure 1A and Supplementary information, Data S1). In brief, feminized fog-2(q71) mutant worms lacking sperm were mated with males expressing a given centriolar protein fused to GFP, and the resulting embryos were analyzed using immunofluorescence with antibodies against GFP and the pan-centriolar marker IFA. Five evolutionarily conserved proteins (SPD-2, ZYG-1, SAS-4, SAS-5 and SAS-6) are specifically required for centriole formation in C. elegans [1], a process that also requires the centriolar constituents $\alpha / \beta$-tubulin $[5,6]$. For our analysis, we selected SAS-4, which has been reported to persist for two cell cycles in C. elegans $[3,4]$, as well as the $\beta$-tubulin protein TBB-2, since centriolar $\alpha / \beta$ tubulin has been shown to persist for one cell cycle in mammalian cells [2]. Moreover, we an- alyzed the behavior of SAS-6, the founding member of a protein family critical for the onset of centriole assembly across eukaryotic evolution $[7,8]$.

These marked mating experiments revealed that centriolar GFP::SAS-4 persisted without significant decrease in signal intensity during the first four embryonic cell cycles (Figure 1B-1D). We likewise assessed the persistence of paternally contributed GFP::TBB-2 and SAS-6::GFP. As shown in Supplementary information, Figure S1A-S1F, these components also persisted in the early embryos without detectable decrease in signal intensity during the first four cell cycles. The SAS-6 data are especially surprising in the light of findings in human cells, where the cartwheel protein HsSAS-6 is degraded during mitosis at each cell cycle [9]. We also noted that the signal intensity of GFP::TBB-2 often differs between the two paternally contributed centrioles (Supplementary information, Figure S1A-S1B), perhaps reflecting a difference in the number of GFP-tagged proteins incorporated into centrioles during spermatogenesis.

We next investigated for how long paternally contributed centriolar components can be detected during embryonic development. To this end, we used both GFP::SAS-4 and SAS-6::GFP, as their stronger signal compared to that of GFP::TBB-2 enabled more robust detection. Remarkably, we found that the two paternal centrioles marked by GFP::SAS-4 could be identified in all embryos up to the $\sim 350$-cell stage (Figure $1 \mathrm{E}$ and $1 \mathrm{G})$. Furthermore, one paternal centriole carrying GFP::SAS-4 could frequently be detected even at the $\sim 550-$ cell stage (Figure 1F and $1 \mathrm{G}$ ). Comparable results were found with SAS-6::GFP (Supplementary information, Figure S1G-1I), although the centriolar GFP signal became undetectable earlier in this case, possibly due to the overall dimmer intensity of this fusion protein compared to GFP::SAS-4. The fact that both GFP::SAS-4 and SAS-6::GFP were no longer detected in the majority of cases towards the end of embryogenesis could reflect technical limitations in detecting centriolar proteins by immunofluorescence at late stages. In support of this notion, we observed that the IFA pan-centriolar staining is 
A

Experimental design:

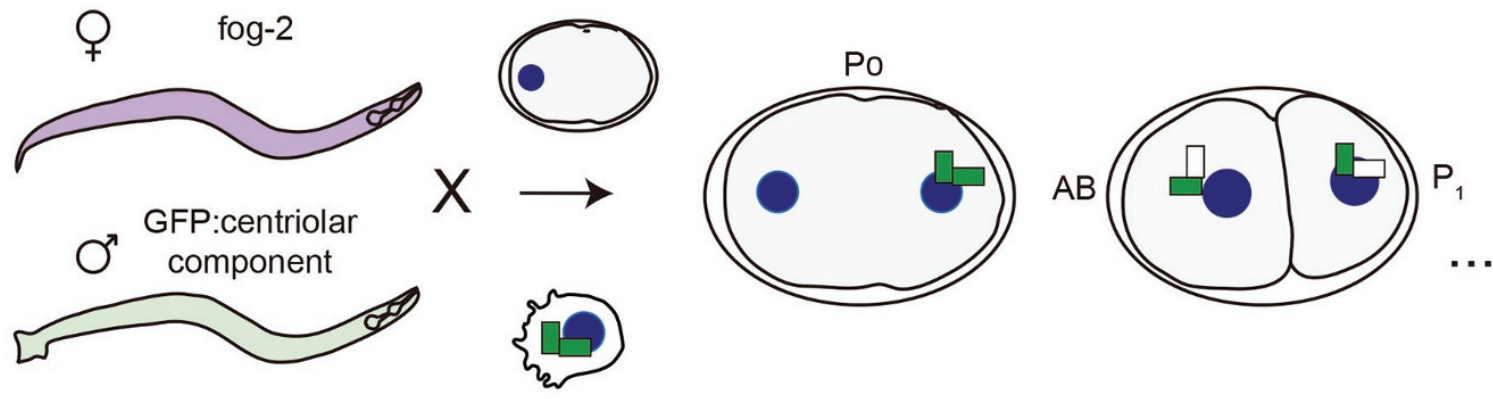

\section{GFP::SAS-4}
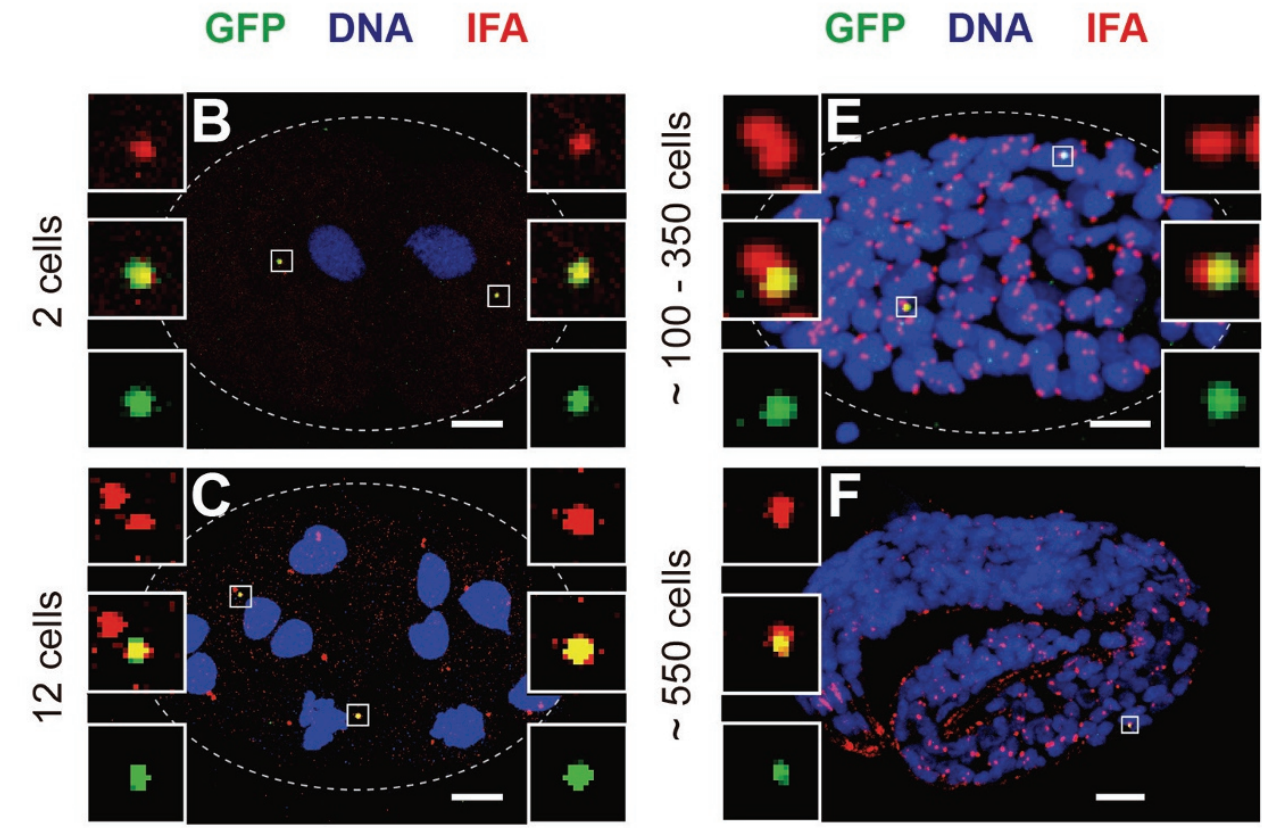

D
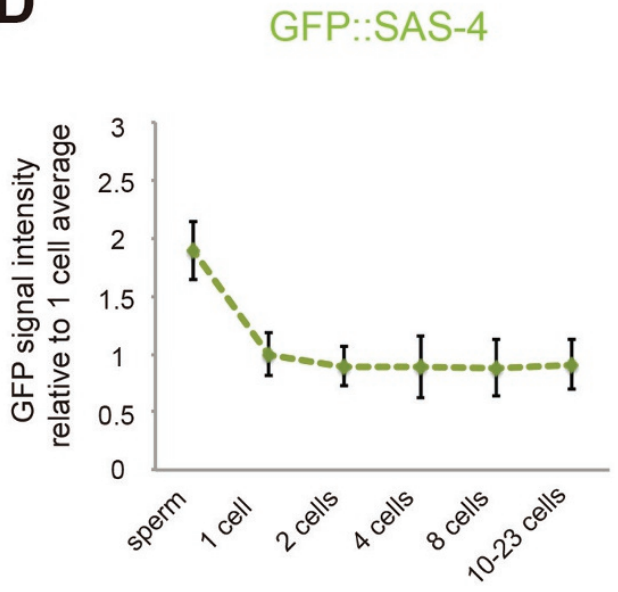

G

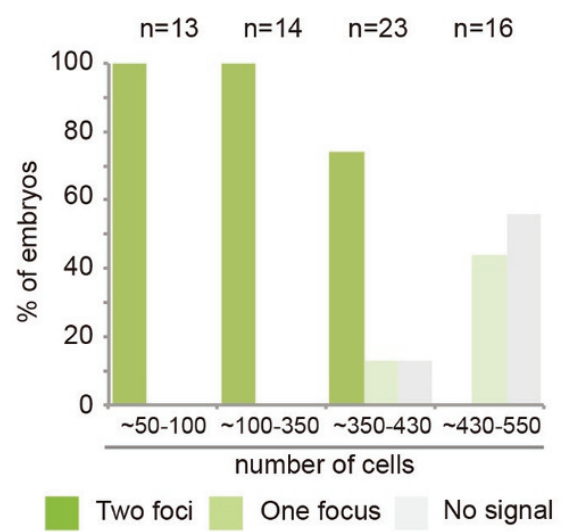


Figure 1 Paternally contributed centriolar components persist throughout $C$. elegans embryogenesis. (A) Marked mating experimental strategy to track paternally contributed centriolar components during C. elegans embryogenesis. Feminized fog-2 animals were crossed with males expressing given GFP-tagged centriolar components in the germline; the zygotes generated thereby inherited paternally contributed GFP-positive centrioles. Thereafter, centrioles formed in the embryo were assembled using the maternally contributed cytoplasmic pool of proteins, which is GFP negative. (B, C, E, F) C. elegans embryos at the indicated stages stemming from marked mating experiments with GFP::SAS-4 males. Embryos were stained with antibodies against GFP (green) and the pan-centriolar marker IFA (red), as well as with Hoechst to reveal DNA (blue). Scale bar, $5 \mu \mathrm{m}$. Insets are 5-time magnified views of centrioles. Note that as a result of unspecific immunostaining, small background green foci not colocalizing with IFA can be observed in some images; such foci were not taken into consideration for analysis. (D) Quantification of the GFP signal of paternally contributed centrioles at the indicated stages of early development for GFP::SAS-4 by immunofluorescence analysis. Signal intensity is expressed relative to the average signal of the 1-cell stage embryos. The approximately two-fold intensity observed in sperm compared to 1-cell stage embryos reflects the fact that sperm cells carry two GFP-tagged centrioles. Number of centrioles analyzed: sperm, $n=417 ; 1$ cell, $n=20$; 2 cells, $n=14$; 4 cells, $n=16$; 8 cells, $n=16$; 10-23 cells, $n=20$. (G) Percentage of embryos with 1, 2 or no GFP-positive paternally contributed centrioles from marked mating experiments with GFP::SAS-4 at the indicated stages of embryogenesis.

less reliable towards the end of embryogenesis (Supplementary information, Figure S1J-S1M).

In summary, our data demonstrate that paternally contributed centriolar components exhibit remarkable persistence in the embryo. Given that $\beta$-tubulin, SAS-4 and SAS-6 are core structural components of centrioles, the entire centriole organelle could be considered to exhibit exceptional persistence across many cell cycles. It will be of interest to determine whether such exceptional persistence can be extended to centrioles of somatic cells in nematodes and also to other systems. Macromolecular complexes other than centrioles exhibit long-term persistence. This is the case for example of nucleopores in terminally differentiated cells, where cumulative damages in some nucleopore constituents have been linked to cell aging, both in C. elegans and rats [10]. Similarly, cumulative damages of centriolar components exhibiting long-term persistence could eventually be detrimental to function and even contribute to disease. Our findings also raise the intriguing possibility that centrioles may act as information carriers across several cell cycles, for instance through posttranslational modifications of the persistent centriolar proteins reported here. Conceivably, this could also allow the paternal gamete to donate dedicated information to the zygote, in a manner that is conceptually analogous and reciprocal to the contribution of maternally provided mitochondria.

\section{Acknowledgments}

We are grateful to Julien Dumont for sharing the strain expressing GFP::TBB-2. For the gift of additional strains, we thank Alex Dammermann and the Caenorhabditis Genetics Center, which is funded by the NIH National Center for Research Resources
(NCRR). We also thank Alexandra Bezler, Marie Delattre and Virginie Hamel for comments on the manuscript. FRB held a fellowship from the Fundación Ramón Areces. This work was funded in part by grants to PG from the ERC (AdG 233335 and AdG 340227).

Fernando R Balestra $^{1}$, Lukas von Tobel ${ }^{1}$, Pierre Gönczy ${ }^{1}$

${ }^{I}$ Swiss Institute for Experimental Cancer Research (ISREC), School of Life Sciences, Swiss Federal Institute of Technology (EPFL) Lausanne, Switzerland

Correspondence: Pierre Gönczy

E-mail: pierre.gonczy@epfl.ch

\section{References}

1 Gönczy P. Nat Rev Mol Cell Biol 2012; 13:425-435.

2 Kochanski RS, Borisy GG. J Cell Biol 1990; 110:1599-1605.

3 Kirkham M, Muller-Reichert T, Oegema K, et al. Cell 2003; 112:575587.

4 Leidel S, Gönczy P. Dev Cell 2003; 4:431-439.

5 Delattre M, Canard C, Gönczy P. Curr Biol 2006; 16:1844-1849.

6 Pelletier L, O'Toole E, Schwager A, et al. Nature 2006; 444:619-623.

7 Leidel S, Delattre M, Cerutti L, et al. Nat Cell Biol 2005; 7:115-125.

8 Dammermann A, Muller-Reichert T, Pelletier L, et al. Dev Cell 2004; 7:815-829.

9 Strnad P, Leidel S, Vinogradova T, et al. Dev Cell 2007; 13:203-213.

10 D'Angelo MA, Raices M, Panowski SH, et al. Cell 2009; 136:284-295.

11 Leung B, Hermann GJ, Priess JR. Dev Biol 1999; 216:114-134.

(Supplementary information is linked to the online version of the paper on the Cell Research website.)

(c) (1)(2)(2) This work is licensed under the CreativeCommons-Attribution-NonCommercial-Share Alike Works 3.0 Unported License. To view a copy of this license, visit http://creativecommons.org/licenses/by-nc-sa/3.0 